\title{
The impact and relationship of inflammatory markers and radiologic involvement in the COVID-19 patients
}

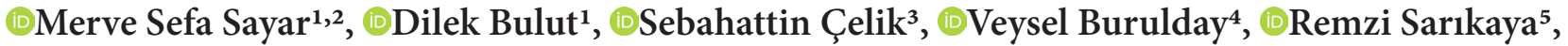 \\ (D) Nurettin Kurt ${ }^{6}$ \\ ${ }^{1}$ Van Training and Research Hospital, Department of Infectious Diseases and Clinical Microbiology, Van, Turkey \\ ${ }^{2}$ University of Health Sciences, Bursa Yuksek Ihtisas Training and Research Hospital, Department of Infectious Diseases and Clinical \\ Microbiology, Bursa, Turkey \\ ${ }^{3}$ Van Training and Research Hospital, Deparment of General Surgery, Van, Turkey \\ ${ }^{4}$ Van Training and Research Hospital, Deparment of Radiology, Van, Turkey \\ ${ }^{5}$ Van Training and Research Hospital, Department of Cardiology, Van, Turkey \\ ${ }^{6}$ Van Training and Research Hospital, Department of Anesthesia and Reanimation, Van, Turkey
}

Cite this article as: Sayar MS, Bulut D, Çelik S, Burulday V, Sarıkaya R, Kurt N. The impact and relationship of inflammatory markers and radiologic involvement in the COVID-19 patients. J Health Sci Med 2021; 4(4): 416-421.

\begin{abstract}
Aim: In the study, it was aimed to investigate the relationship between inflammatory markers and radiology in COVID-19 patients.

Material and Method: The study was conducted in the quarantine wards of a tertiary hospital between March and May 2020. Patients with a definite diagnosis of COVID-19 were included in the study. The lung damage of the patients caused by COVID-19 was determined by computed tomography and the relationship between lung damage and inflammatory markers was examined.

Results: The mean age of 259 COVID-19 patients included in the study was $61.96 \pm 14.076$. Except for thrombocytopenia, all variables such as ferritin, D-dimer, thoracic computerized tomography $(\mathrm{CT})$ involvement rates were significantly poorer in the patients requiring the care in ICU than the patients in wards $(\mathrm{p}<0.001)$. No chronic disease was found in $193(74.5 \%)$ of 259 patients. In multi-variate analyzes, elderly and high thoracic CT involvement rate were determined as independent variables determining the serious disease risks and are important parameters in assessing the need for ICU ( $\mathrm{p}<0.05)$. Ferritin value, D-Dimer value on the third day of admission, Neutrophil lymphocyte ratio and leukocyte count were found to be correlated with thoracic CT involvement rate $(\mathrm{p}<0.05)$.

Conclusion: It was observed that there were serious changes in the infection parameters of COVID-19 cases with advanced radiological involvement in the lung.
\end{abstract}

Keywords: COVID-19, intensive care unit, prognosis, SARS-CoV-2

\section{INTRODUCTION}

In the last 10 years, infectious diseases have posed an ongoing threat, and especially acute respiratory viruses are among the most recent and re-emerging infectious diseases due to the burden led by severe disorders. Human metapneumo virus (described first in 2001), SARS-related coronavirus (described in 2003), human bocavirus (described in 2005), pandemic influenza A (H1N1) (described in 2009) and the novel Middle Eastern respiratory syndrome coronovirus (MERS-CoV) associated with severe respiratory disease in 2012 are among the examples of acute respiratory infections in the $21^{\text {st }}$ century (1). The novel coronavirus 2019 pneumonia
(COVID-19) led by severe acute respiratory syndrome coronavirus (SARS-CoV-2) was detected for the first time in December 2019 in the province of Wuhan, China and has become one of the fatal outbreaks of respiratory infections in the $21^{\text {st }}$ century. To date, 57.8 million cases and 1.3 million deaths have been witnessed worldwide since the outbreak of the COVID-19 (2). The worries of an efficiency antiviral agent and vaccine against the virus with high infectivity and the existence of an asymptomatic population are the factors complicating the therapeutic management of COVID-19 (3). As well as the asymptomatic or mild upper respiratory symptoms, some 
cases of SARS-CoV-2 are encountered with severe course of pneumonia, characterized by fever, cough, dyspnea, bilateral pulmonary infiltration and acute respiratory disease (4). A study stated that approximately $19 \%$ of the cases progressed to severe respiratory disease, and a mortality rate of $2.3 \%$ was reported (5). Since a clinically effective drug targeting directly the virus in the cases of SARS-CoV-2 has yet to be created, it is important to identify the cases where COVID-19 is likely to progress critically or severely (6). In the study, it was aimed to examine the factors affecting the severity of the disease in COVID-19 cases, as well as to examine the relationship between infection parameters and radiology.

\section{MATERIAL AND METHOD}

An approval was obtained from the Ethical Board of Van Training and Education Hospital (Date: 18/06/2020, Decision No: 2020/11). All procedures were carried out in accordance with the ethical rules and the principles of the Declaration of Helsinki.

\section{Patients and Design}

Of 259 patients who were diagnosed with definitive COVID-19 and followed-up in the quarantine hospitals in the province of Van between $1^{\text {st }}$ March and $15^{\text {th }}$ May 2020, were included into the study. The definitive diagnosis of COVID-19 infection was made by detecting the positivity ofCOVID Real-time polymerase chain reaction (RT-PCR) in the samples upper respiratory tract (nasopharyngeal and oropharyngeal swabs) and, if possible, lower respiratory tract (sputum, tracheal aspirate, or broncho alveolar lavage) of patients in quarantine hospitals. The patients were evaluated clinically, radiologically and in terms of laboratory tests, and those with definitive diagnosis of COVID-19 showing RT-PCR positivity in recurrent tests were included into the study (at least one of them was positive). The patients who are under 16 years of age and considered to be with COVID-19 based on the clinical, laboratory and radiologic investigations, and those without RT-PCR positivity in the repetitive samples from upper or lower respiratory tracts (If no COVID RT-PCR positivity is detected in at least one of the two samples) were excluded out of the study. Patients who were found to be positive in at least two COVID RT-PCR tests were being diagnosed with COVID-19 and included in the study. The parameters of the patients diagnosed with COVID-19 such as age, gender, history of additional diseases, initial time of complaints, length of hospital stay, length of staying in ICU, fever, saturation, respiratory rate, and patients' laboratory and radiological findings were acquired retrospectively from the patients discharge and hospital medical records. Radiological imaging was not performed in cases with pregnancy and in cases where radiological imaging was not required by the clinician. According to the severity of the disease, the need for intensive care was decided with the following criteria:
- Those with dispnea and respiratory distress,

- Respiratory rate $\geq 30 /$ minute,

- Arterial oxygen partial pressure $\left(\mathrm{PaO}_{2}\right) /$ fractional inspired oxygen $\left(\mathrm{FiO}_{2}\right)<300$,

- Those requiring oxygen during the follow-up,

- Those with serum pressure oxygen-2 $\left(\mathrm{SpO}_{2}\right)<90 \%$ or $\mathrm{PaO}_{2}<70 \mathrm{mmHg}$ despite oxygen treatment of $5 \mathrm{~L} / \mathrm{min}$,

- Hypotension [systolic blood pressure (SBP) $<90$ $\mathrm{mmHg}$, a decrease more than $40 \mathrm{mmHg}$ of normal SBP, mean arterial pressure $<65 \mathrm{mmHg}$ and tachycardia $>100 / \mathrm{min}]$

- Those with the development of immunosuppression and acute organ dysfunction, such as acute kidney injury, impairments in acute liver function tests, confusion and acute hemorrhagic diathesis

- High levels of troponin and arrhythmia

- Lactate $>2$ mmol (7)

Only patients hospitalized in a quarantine hospital were included in the study. Antiviral and antimicrobial treatments were applied to all patients here. In addition, supportive treatments (such as anticoagulants, immunomodulators or corticosteroids) were applied according to the laboratory and clinical conditions of the patients. Treatment modalities were personalized for each patient according to the presence of contraindications, probable development of drug-drug interactions and toxicity.

In COVID-19 cases with low saturation and increased respiratory rate accompanied by high D-Dimer, the differential diagnosis of "Pulmonary Thromboembolism" (PTE), was made by pulmonologists and cardiology physicians (the differential diagnosis of "Pulmonary Thromboembolism" (PTE). In addition to these, pulmonary angiography and echocardiography were performed by pulmonologists and cardiology physicians.

\section{Laboratuary Assessment}

Complete blood count, biochemical analysis, coagulation tests, renal function tests, $\mathrm{C}$-reactive protein (CRP), ferritin and $\mathrm{D}$-dimer parameters of all patients on admission and on their third day of antiviral therapy were evaluated. Lymphopenia was defined as lymphocyte count $<800$ cells $/ \mathrm{mm}^{3}$. Thrombocytopenia was defined platelet count $<150,000 / \mathrm{mm}^{3}$ respectively.

\section{Radiologic Analysis}

Each scanning was obtained in the supine position, and no contrast and sedation were used for the procedure. The images were acquired with a 16-slice CT (MSCT; Siemens X-Ray Vacuum Technology, Jiangsu, China) and using the following parameters: Tube voltage, $130 \mathrm{kV}$; effective $\mathrm{mAs}, 25$; slice thickness, $5 \mathrm{~mm}$; scanning field of the view, $350 \mathrm{~mm}$; and image matrix, $512 \times 512$. Also, 
the images were taken at the axial plane. Thoracic CT images were evaluated retrospectively on a PACS work station with multiplanar reconstruction by one radiologist (VB) having 10-year experience in interpreting thoracic CT images. A semi-quantitative scoring system was used to quantitatively estimate the pulmonary involvement of all these abnormalities on the basis of the area involved. In order to quantify the extent of lesions, CT score was assigned on the basis of all abnormal areas involved. Each lobe was assigned a score based on the following criteria: Score 0, 0\% involvement; score 1, less than 5\% involvement; score 2, 5 to $25 \%$ involvement; score 3, 26 to $49 \%$ involvement; score 4, 50 to $75 \%$ involvement; and score 5 , greater than $75 \%$ involvement. The total CT score was the sum of the individual lobar scores and ranged from 0 (no involvement) to 25 (maximum involvement) $(8,9)$. After each patient was scored on CT, a six-graded staging criterion was constituted according to total chest CT involvements as follows:

Grade 0: 0 (no involvement)

Grade 1: 0-5 (mild)

Grade 2: 6-10 (mild-to-moderate)

Grade 3: 11-15 (moderate)

Grade 4: 16-20 (severe)

Grade 5: >20 (advanced severe)

\section{Statistical Analysis}

The statistical analyzes of the data obtained in the study were carried out using the Standard Package for Social Sciences for Windows, version 18.0 (SPSS, Chicago, IL, USA). For the comparison between the groups, the student $\mathrm{t}$-test was used, and a $\mathrm{p}$ value less than 0.05 was considered to be significant. Relationships with categorical variables such as gender of patients in ICU and in ward, were examined. While the "enter" method was used for the variables seen to be significant in the univariate analyzes, the multivariate analyzes were performed using the "forward conditional" method.

\section{RESULTS}

General characteristics of the patients: It was determined that 24 of 259 patients followed up within the scope of the study needed to be followed in the intensive care unit. The average age level of 24 patients with severe COVID-19 in ICU was determined to be $61.96 \pm 14.076$ years. It was observed that the patients followed up in ICU were older ( $\mathrm{p}<0.05)$, (Table 1). In multivariate analyzes, advanced age was determined as an independent variable in severe COVID-19 cases $(\mathrm{p}<0.05)$ (Table 2). In terms of gender differences, It was observed that the patients with severe COVID-19 were $8.4 \%$ male patients. When the relationship between gender and ICU need was examined, it was observed that there was no significant relationship ( $p>0.005)$. Thirty-four (13.1\%) patients were determined to have at least one chronic disease while $19(7.33 \%)$ had two chronic diseases. The distribution of chronic diseases was determined as hypertension $15.4 \%(\mathrm{n}=40)$, diabetes mellitus (DM) 9.2\% $(\mathrm{n}=24)$ and chronic lung disease $7.72 \%$ $(n=20)$. No significant relationship was found between hypertension and the requirements of ICU $(\mathrm{p}>0.005)$. While 95.7\% (248/259) of the patients in the study were discharged from the hospital, it was observed that $4.2 \%$ $(11 / 259)$ of them died. All of the cases that resulted in death were being followed up in the ICU.

\begin{tabular}{|c|c|c|c|c|}
\hline \multicolumn{2}{|l|}{ Variables } & $\begin{array}{l}\text { Inpatients in wards } \\
\quad(n=235)\end{array}$ & $\begin{array}{c}\text { ICU patients } \\
(n=24)\end{array}$ & $\mathbf{p}$ \\
\hline \multicolumn{2}{|c|}{ Age mean \pm SD (years) } & $38.37 \pm 16.593$ & $61.96 \pm 14.076$ & $<0.001$ \\
\hline \multirow{2}{*}{ Gender } & Male & $131(\% 91.6)$ & $12(\% 8.4)$ & \multirow{2}{*}{0.590} \\
\hline & Female & $104(\% 89.7)$ & $12(\% 10.3)$ & \\
\hline \multirow{7}{*}{$\begin{array}{l}\text { Clinical } \\
\text { parameters }\end{array}$} & Fever $\left({ }^{\circ} \mathrm{C}\right)$ & $36.5 \pm 0.74$ & $37.2 \pm 0.98$ & $\mathrm{P}=0.005$ \\
\hline & Respiratory rate/minute & $19.2 \pm 2.68$ & $31.8 \pm 7.08$ & $<0.001$ \\
\hline & Oxygen saturation (\%) & $95.2 \pm 4.68$ & $77.08 \pm 15.4$ & $<0.001$ \\
\hline & qSOFA values & & & \multirow{4}{*}{$<0.001$} \\
\hline & 0 & $217 \pm 99.1$ & $2 \pm 0.9$ & \\
\hline & 1 & $17 \pm 50.0$ & $17 \pm 50.0$ & \\
\hline & 2 & $1 \pm 16.7$ & $5 \pm 83.3$ & \\
\hline \multirow{10}{*}{$\begin{array}{l}\text { Laboratory } \\
\text { parameters }\end{array}$} & Leukocyte (/L) & $6153.48 \pm 2507.48$ & $8774.58 \pm 4610.43$ & $<0.001$ \\
\hline & Lymphocyte $<800$ cells per $\mathrm{mm}^{3}$ & $1613 \pm 615.44$ & $1070 \pm 408.28$ & $<0.001$ \\
\hline & Platelets $<150.000$ per $\mathrm{mm}^{3}$ & $216697.87 \pm 57445.758$ & $195916.67 \pm 49400.684$ & 0.089 \\
\hline & NLR & $2.98 \pm 2.32$ & $7.66 \pm 6.1$ & $<0.001$ \\
\hline & CRP (mg/L) & $13.68 \pm 29.85$ & $101.68 \pm 96.02$ & $<0.001$ \\
\hline & Ferritin value on admission & $169.55 \pm 201.104$ & $493.2 \pm 434.51$ & $<0.001$ \\
\hline & Ferritin value on $3^{\text {rd }}$ day of antiviral treatment & $182.44 \pm 248.42$ & $615.12 \pm 544.97$ & $<0.001$ \\
\hline & D-dimer value on admission $(\mathrm{ng} / \mathrm{mL})$ & $261.11 \pm 632.66$ & $1192.59 \pm 3271.75$ & $<0.001$ \\
\hline & D-dimer value on $3^{\text {rd }}$ day of antiviral treatment $(\mathrm{ng} / \mathrm{mL})$ & $273.15 \pm 757.08$ & $965.3 \pm 1016.45$ & $<0.001$ \\
\hline & Creatine $(\mathrm{mg} / \mathrm{dL})$ & $0.93 \pm 0.49$ & $1.26 \pm 0.7$ & $<0.001$ \\
\hline \multicolumn{2}{|c|}{ Radiologic parameters (according to involvement rates) (\%) } & $7.56 \pm 13.09$ & $50.48 \pm 29.2$ & $<0.001$ \\
\hline
\end{tabular}


Table 2. Analysis of independent variables through the Binary Logistic Regression in the development of severe COVID-19

\begin{tabular}{|lccc|}
\hline Independent variables & OR & $\mathbf{9 5 \%}$ CI & $\mathbf{p}$ \\
\hline Advanced age & 1.07 & $1.0-1.13$ & $\mathrm{p}=0.036$ \\
Thorax CT involvement rate & 1.06 & $1.02-1.09$ & $\mathrm{p}<0.001$ \\
\hline CI: Confidence interval, CT: Computerized tomography, OR: Odds ratio & \\
\hline
\end{tabular}

Laboratory characteristics of the patients: The mean leukocyte count in the patient group in need of ICU was $8774.58 \pm 4610.43$ and the leukocyte value was statistically significantly higher in this group $(\mathrm{p}<0.05)$. It was observed that there was a significant increase in lymphopenia, CRP, NLR, D-Dimer, and Creatine values except thrombocytopenia in patients in the ICU group ( $p<0.05)$. All the variables of the patients requiring to be cared in ICU, except for thrombocytopenia, were significantly different from those of the inpatients followed-up in wards $(\mathrm{p}<0.001)$, and COVID-19 patients with the requirements of ICU were found to have poorer values (Table 1). The mean ferritin values of all patients were detected to be $199.47 \pm 249.48$ on admission and $223.93 \pm 315.15$ on 3rd day of antiviral therapy. $\mathrm{D}$-dimer values of all patients were also seen as $345.79 \pm 1169.81$ on admission and $343.9 \pm 812.37$ on $3^{\text {rd }}$ day of antiviral therapy. In addition, the average rates of neutrophil/lymphocyte ratio (NLR) and leukocyte were observed to be $3.45 \pm 3.24$ and $6396.37 \pm 2859.6$ on admission, respectively. In $11.7 \%(30 / 257 * 100)$ of the 257 patients included in the study, their first RT-PCR results were negative. RT-PCR tests performed with repeated in the repetitive samples from nasopharyngeal and oropharyngeal swabs were found positive on the $2-3^{\text {rd }}$ day of the follow-up in the quarantine hospital of the patients whose first RT-PCR test was negative.

The relations of inflammatuary markers and radiological involvement: No radiological imaging was performed for 38 of 259 participants since five were pregnant, and 33 were assessed not to require the imaging by the clinician. The rate of total thoracic CT involvements in 221 patients undergoing thoracic CT was found as $11.6 \pm 19.8$. However, when the rate of lung involvements was examined, no involvement was observed in the lung parenchyma in $57 \%(n=126)$ of the cases. It was seen that $7 \%(15 / 221)$ of all patients with thoracic CT scan had parenchymal involvement over $50 \%$ (Table 3). As the rate of thoracic CT involvements increased, a positive increase was also observed in almost all parameters. The parameter demonstrating the best correlation with the rate of lung involvements was found in ferritin (52\% positively significant). While there was no correlation between D-Dimer value and the rate of thoracic CT involvements on admission, a weak correlation of $27 \%$ was observed between them on the 3rd day of treatment and this correlation was found to be significant (Table 4). It could be considered that as the lung involvements increase, D-Dimer value will increase, or vice versa. Moreover in multivariate analyzes, involvement rate was determined as an independent variable in severe COVID-19 cases $(\mathrm{p}<0.05)$ (Table 2).

\begin{tabular}{|lcc|}
\hline \multicolumn{3}{|l}{$\begin{array}{l}\text { Table 3. Distribution of the involvement rates in Thorax CT of the } \\
\text { cases }\end{array}$} \\
$\begin{array}{l}\text { The involvement rates } \\
\text { of cases in thorax CT }\end{array}$ & $\begin{array}{c}\text { Number of } \\
\text { patients (n:) }\end{array}$ & Percent (\%) \\
\hline 0 & 126 & 57.0 \\
4 & 5 & 2.3 \\
8 & 20 & 9.0 \\
12 & 4 & 1.8 \\
16 & 17 & 7.7 \\
20 & 10 & 4.5 \\
24 & 5 & 2.3 \\
28 & 4 & 1.8 \\
32 & 3 & 1.4 \\
36 & 5 & 2.3 \\
40 & 4 & 1.8 \\
44 & 1 & 0.5 \\
48 & 2 & 0.9 \\
56 & 3 & 1.4 \\
64 & 3 & 1.4 \\
68 & 1 & 0.5 \\
72 & 3 & 1.4 \\
76 & 1 & 0.5 \\
80 & 2 & 0.9 \\
92 & 2 & 0.9 \\
Total & $\mathbf{2 2 1}$ & $\mathbf{1 0 0 . 0}$ \\
\hline
\end{tabular}

\begin{tabular}{|lcc|}
\hline \multicolumn{3}{|c|}{ Table 4. Correlation of radiological tutulum with each other } \\
\hline & $\begin{array}{c}\text { Correlation } \\
\text { coefficient } \\
\mathbf{r}_{\mathbf{s}}\end{array}$ & $\mathbf{P}$ \\
\hline $\begin{array}{l}\text { Ferritin value on admission- Total } \\
\text { involvement }\end{array}$ & 0.521 & $0.00^{*}$ \\
$\begin{array}{l}\text { Ferritin value on 3 } \\
\text { treatment- Total involvement }\end{array}$ & 0.422 & $0.00^{*}$ \\
$\begin{array}{l}\text { D-dimer value on admission- Total } \\
\text { involvement }\end{array}$ & 0.042 & $0.546^{*}$ \\
$\begin{array}{l}\text { D-dimer value on 3 } \\
\text { treatment- Total involvement }\end{array}$ & 0.270 & $0.000^{*}$ \\
$\begin{array}{l}\text { NLR- Total involvement } \\
\text { Leukocyte count- Total involvement }\end{array}$ & 0.406 & $0.000^{*}$ \\
\hline NLR: Neutrophil/lymphocyte ratio & 0.295 & $0.000^{*}$ \\
\hline
\end{tabular}

\section{DISCUSSION}

The patients diagnosed with COVID-19 are encountered in a wide spectrum from asymptomatic cases to severe pneumonia and even death. COVID-19 cases were reported in the WHO-China joint report in February 2020; Eighty percent of those with a definite diagnosis thanks to the laboratory tests have mild or moderate disease (with or without pneumonia), 13.8\% experience the disease severely (dyspnea, breath count $\geq 3 / \mathrm{min}$, blood oxygen saturation $\leq 93 \%, \mathrm{PaO}_{2} / \mathrm{FiO}_{2}$ ratio $<300$, 
and / or lung infiltration of $>50 \%$ between $24-48$ hours) and $6.1 \%$ also have critical disease (respiratory failure, septic shock, and / or multiple organ dysfunction / failure) (10). The requirements of ICU are seen in 5\% of all COVID-19 patients (11). The presence of comorbid factors, such as male gender, over 60 years of age, hypertension, DM, cerebrovascular, cardiovascular and chronic respiratory diseases, and cancers are among the risk factors leading to severe COVID-19 pneumonia (12-14). In the study, it was seen that 9.2\% (24/259) of the patients needed intensive care. Similar to the data in other studies, COVID-19 was found to have a more severe prognosis in advanced aged patients in our study ( $p<0.05)$. It was determined that the most common accompanying chronic disease was hypertension $(15.4 \%$, $(n=40))$. However, there was no significant relationship between the severity of the disease ( $p>0.05)$.

Metabolic diseases cause the immunal functions to be reduced by leading to lymphocyte and macrophage dysfunction, as well as the patient to be more sensitive to the complications of COVID-19 disease (15). During COVID-19 infection, both the innate and acquired immune system participates synergistically in the antiviral response, and a significant increase is seen in leukocytes, neutrophils and NLR, along with significant lymphopenia in severe cases (16). However, in the study in which 225 patients were investigated by $\mathrm{Li}$ et al., it was stated that $37(16.44 \%)$ patients were diagnosed with severe COVID-19 disease, and patients' leukocyte (86.67\%) and lymphocyte $(99.11 \%)$ counts were within normal levels or decreased on admission, and the levels of CRP (mean, 60.4 \pm 57.5 ; normal range, $0-10 \mathrm{mg} / \mathrm{L}$ ), procalcitonin $(0.87 \pm 0.56$; normal range, $0-0.5 \mathrm{mg} / \mathrm{L})$ and sedimentation (mean, 55.8 \pm 25.3 ; normal range, 0-15 $\mathrm{mm} / \mathrm{h}$ ) were increased in $86.22,10.67$ and $90.22 \%$ of the patients, respectively (17). In another study by Guan et al., however, lymphocytopenia, thrombocytopenia and leukopenia were detected to be present in 83.2, 36.2 and $33.7 \%$ of the patients on admission respectively, and the levels of CRP, alanine amino transferase (ALT), aspartate amino transferase (AST), creatine kinase (CK) and D-dimer were found to be higher in general (18). In the same study, it is reported that $61.1 \%$ of the patients with severe COVID-19 presented with leukopenia, $96.1 \%$ with lymphopenia (below 1500 per $\mathrm{mm}^{3}$ ) and $57.7 \%$ with thrombocytopenia, while the levels of CRP, procalcitonin and $\mathrm{D}$-dimer were found higher as 81.5 , 13.7 and $59.6 \%$, respectively (18). As can be seen in the mentioned studies; In COVID-19 cases, it is known that comorbid factors as well as laboratory changes are associated with a more severe clinical course. When the laboratory parameters of the cases in our study are examined; Patients with increased levels of leukocytes, NLR, CRP, ferritin, D-dimer and creatinine and those with lymphopenia were found to be significantly more severe patients $(\mathrm{p}<0.001)$. These data obtained in the study have added strength to the literature in this respect.

Patients with severe prognosis were found to be worse in the laboratory when evaluated both clinically and laboratory. The laboratory and clinical data of the patients in our study were found to support other studies in this area, and in this respect, it was necessary to examine the relationship between the laboratory and the radiological evaluations of patients. As known; the investigations through thorax CT imaging as an important modality in the diagnosis of lung diseases are of vital sensitivity in the diagnosis and evaluation of COVID-19 disease (19). Under The radiological imaging guide released by the World Health Organization (WHO), it is recommended that the need for imaging be supported by the patient's clinic, and the laboratory and RT-PCR results (20). As thorax CT findings can be detected as normal in half of the patients within the first 1-2 days after the symptoms begin, radiological findings can also be detected even before the symptom starts $(19,21)$. Bilateral lung involvement develops in over $75 \%$ of thorax CT images in COVID-19 cases, and multilobar involvement is frequently observed (71\%) (22). Among the most common finding in thorax CT images are ground glass opacities, and ground glass opacities were observed in varying rates between $86-77 \%$ in thorax CT series (2123). In the study where Shi et al. examined the rate of radiological involvement in terms of weeks, leukocytosis (32\%) and lymphocytosis (67\%) were found to be high, as well as CRP concentration and serum amyloid A protein levels, in most of the patients (24). Ayrica In the study by Zhang et al., as consistent with the findings reported in other studies, the increase in $\mathrm{D}$ - dimer was found to be statistically significant in predicting mortality (25). As it can be seen, most COVID-19 cases come with the presence of diffuse lung involvement. It is also known that there is a significant relationship between lung involvement and the laboratory. In our study, we found that the average of thorax CT involvements was found to be $50.48 \pm 29.2$ and also, as the lung involvement rate increased, the severity of the disease increased.

Laboratory findings are of great importance in severe COVID-19 cases. In addition to the clinic, radiological progress should be evaluated with the laboratory. It is now well known and supported by many studies that many inflammation indicators such as ferritin, CRP, D-Dimer, NLR are associated with the severity of the disease. In our study, the relationship between ferritin, D-Dimer, Leukoid count and NLR and radiological involvement rates were examined. As a result the value of ferritin on admission was found to be correlated positively and significantly at the rate of $52 \% .27 \%$ correlation was found between 
D-Dimer values on 3rd day of antiviral therapy and rate of thoracic CT involvements. In the light of the data obtained in the study; It would be appropriate to say that serial measurements of ferritin and D-Dimer values in COVID-19 cases will significantly contribute to clinical findings in evaluating the progression of the disease.

\section{CONCLUSION}

COVID-19 cases present with different clinical pictures from asymptomatic to serious. In this respect, clinical and laboratory are of great importance in evaluating the severity of the disease. However, when examining laboratory parameters, the evaluation of patients' inflammatory markers becomes more important than other parameters. In particular the ferritin and D-dimer parameters should be meticulously evaluated on admission and 3rd day of the antiviral treatment, and predicting the rates of lung involvements will be effective in understanding the extent of disease severity.

\section{ETHICAL DECLARATIONS}

Ethics Committee Approval: An approval was obtained from the Ethical Board of Van Training and Education Hospital (Date: 18/06/2020, Decision No: 2020/11).

Informed Consent: Because the study was designed retrospectively, no written informed consent form was obtained from patients.

Referee Evaluation Process: Externally peer-reviewed.

Conflict of Interest Statement: All authors also declare no conflict of interest.

Financial Disclosure: The authors declared that this study has received no financial support.

Author Contributions: All of the authors declare that they have all participated in the design, execution, and analysis of the paper, and that they have approved the final version.

\section{REFERENCES}

1. Khabbaz R, Beth BP, Schuchat A, et al. Emerging and reemerging infectious disease threats. In: Mandel, Dougles, and Bennett's Principles and Practice of Infectious Disease, Bennett JE, Dolin R, Blaser MJ (eds). 8rd ed. Philadelphia: Elsevier; 2015: 158-77.

2. World Health Organization: COVID-19 Weekly Epidemiological Update-24 November 2020. Available at: <https://www.who. int/publications/m/item/weekly-epidemiological-update---24november-2020> Accessed November 25, 2020.

3. Cao W, Li T. COVID-19: towards understanding of pathogenesis. Cell Res 2020; 5: 367-9.

4. Shi Y, Yu X, Zhao H, et al. Host susceptibility to severe COVID-19 and establishment of a host risk score: Findings of 487 cases outside Wuhan. Crit Care 2020; 1:2-5.

5. Wu Z, McGoogan JM. Characteristics of and important lessons from the coronavirus disease 2019 (COVID-19) outbreak in China. JAMA 2020; 13: 1239-42.
6. Zhai P, Ding Y, Wu X, et al. The epidemiology, diagnosis and treatment of COVID-19. Int J Antimicrob Agents 2020; 5: 105955.

7. TR Ministry of Health, General Directorate of Public Health. COVID-19 (SARS-CoV-2 Infection) Treatment of Adult Patients 19 June 2020. Available at: <https://covid19bilgi.saglik.gov.tr/depo/ rehberler/covid-19-rehberi/COVID-19_REHBERI_ERISKIN_ HASTA_TEDAVISI.pdf $>$. Accessed July 1, 2020.

8. Pan F, Ye T, Sun P. Time course of lung changes at chest ct during recovery from coronavirus disease 2019 (COVID-19). Radiology 2020; 295: 715-21.

9. Li K, Wu J, Wu F, et al. The clinical and chest ct features associated with severe and critical COVID-19 pneumonia. Invest Radiol 2020; 6: 327-31.

10. China-WHO Expert team . Report of the WHO-China Joint Mission on Coronavirus Disease 2019 (COVID-19). Available at <https:// www.who.int/docs/default-source/coronaviruse/who-china-jointmission-on-covid-19-final-report.pdf > . Accessed by July 1,2020.

11. World Health Organization: Clinical Care Severe Acute Respiratory Infection: Tool kit. Available at: < https://apps.who.int/iris/bitstream/ handle/10665/331736/WHO-2019-nCoV-SARI_toolkit-2020.1-eng. pdf? sequence $=1$ \&isAllowed $=y>$. Accessed by July 1, 2020 .

12. Harapan H, Itoh N, Yufika A, et al. Coronavirus disease 2019 (COVID-19) : A literature review. J Infect Public Health 2020; 5: $667-73$.

13. Sohrabi C, Alsafi Z, O'Neill N, et al. World Health Organization declares global emergency: A review of the 2019 novel coronavirus (COVID-19). Int J Surg 2020; 76: 71-6.

14. Shi Y, Wang G, Cai XP, et al. An overview of COVID-19. J Zhejiang Univ Sci B 2020; 5: 343-60.

15. Yang J, Zheng Y, Gou X, et al. Prevalence of comorbidities and its effects in patients infected with SARS-CoV-2: a systematic review and meta-analysis. Int J Infect Dis 2020; 94: 91-5.

16. Tufan A, Avanoğlu Güler A, Matucci-Cerinic M. COVID-19, immune system response, hyperinflammation and repurposing antirheumatic drugs. Turk J Med Sci 2020; 50: 620-32.

17. Shi H, Han X, Jiang N, et al. Radiological findings from 81 patients with COVID-19 pneumonia in Wuhan, China: a descriptive study. Lancet Infect Dis 2020; 4: 425-34.

18. Li R, Tian J, Yang F, et al. Clinical characteristics of 225 patients with COVID-19 in a tertiary hospital near Wuhan, China. J Clin Virol 2020; 127: 104363 .

19. Guan W, Ni Z, Hu Y, et al. Clinical characteristics of coronavirus disease 2019 in China. N Engl J Med 2020; 18: 1708-20.

20. Zhang L, Yan X, Fan Q, et al. D-dimer levels on admission to predict in-hospital mortality in patients with Covid-19. J Thromb Haemost 2020; 6: 1324-9.

21. Kamps BS, Hoffmann C (editors). Diagnostic Tests and Prosedures. In: COVID reference. Fourth ed., Hamburg: Steinhauser-Verlag; 2020: 155-85.

22. World Health Organization: Use of chest imaging in COVID-19. Available at: https://www.who.int/publications/i/item/use-of-chestimaging-in-covid-19. Accessed by July 1, 2020.

23. Salehi S, Abedi A, Balakrishnan S, Gholamrezanezhad A. Coronavirus Disease 2019 (COVID-19): A systematic review of imaging findings in 919 patients. AJR Am J Roentgenol 2020; 1: 87-93.

24. Lai CC, Shih TP, Ko WC, Tang HJ, Hsueh PR. Severe acute respiratory syndrome coronavirus 2 (SARS-CoV-2) and coronavirus disease-2019 (COVID-19): The epidemic and the challenges. Int J Antimicrob Agents 2020; 3: 105924.

25. Song F, Shi N, Shan F, et al. Emerging 2019 novel coronavirus (2019$\mathrm{NCoV}$ ) pneumonia. Radiology 2020; 1: 210-7. 